\title{
A COMPUTATIONAL FRAMEWORK FOR MODELING IRRADIATION CREEP AND SWELLING IN SINGLE CRYSTAL NICKEL
}

\author{
Richard W. SMITH * and Gary S. WAS ** \\ The University of Michigan, Department of Nuclear Engineering, Ann Arbor, MI 48109, USA
}

Received 9 July 1985; accepted 13 February 1986

\begin{abstract}
A computational framework for modeling irradiation creep and swelling is developed to estimate creep strains, swelling strains and the dose dependent evolution of microstructural characteristics such as dislocation density, void number density and void size in pure, single crystal metals. This work represents an attempt to construct a comprehensive and self-consistent description of the physical processes that occur during the application of temperature, stress, and irradiation, in that cross-talk between individual microstructural models is allowed during their mutual evolution in time. The coupling of microstructural evolution to the strain generating models is responsible for the following results: the variation of the steady state creep rate with dose in the early part of the irradiation history; the observation that irradiation creep displays a maximum in temperature and falls off at high temperature, similar to swelling; the linear dependence of steady state creep on stress with a discontinuity at $\sigma=0$ resulting from the influence of stress on the void number density. The major violation of self-consistency is the isolation of void nucleation from the microstructural evolution. The magnitudes of creep strains computed with the model compare favorably with measured values for nickel.
\end{abstract}

\section{Introduction}

Virtually all reactor components exhibit some degree of macroscopic deformation during irradiation. Structures such as fuel cladding, control rod guide tubes and BWR assembly wrappers have all been found to experience strains large enough to seriously degrade their performance. In order to account for these strains in the design of reactor components, designers and modelers use empirical correlations which relate the extent of deformations to the controlling parameters such as stress, temperature, and neutron fluence.

Empirical models describing irradiation creep and void swelling for various materials are abundant in the literature. The utility of these models is, however, greatly limited because they do not account for variations in many of the key parameters such as initial dislocation density or displacement rate. It is therefore impossible to extrapolate the known correlations to different conditions with any degree of confidence. In order to facilitate such an extrapolation it is necessary to develop "mechanism-based" models of the strain produc-

\footnotetext{
* Graduate Student.

** Associate Professor.
}

ing processes. These models will provide physically justifiable methods of extrapolating empirical correlations and identifying key parameters. In addition, mechanism-based models will serve as an important means of verifying theoretical descriptions of various microstructural processes.

Many mechanism-based models of irradiation creep and swelling processes have been presented over the past several years [1-10]. However, modeling the microstructural evolution of a solid under irradiation requires that the individual process models be combined to form a "macro" model. A fundamental problem common to each of these models is a lack of self-consistency and completeness with respect to the evolution of microstructure. That is, the individual models do not properly account for or recognize each others' existence. Processes such as the generation and annihilation of dislocations are usually ignored and the response of each process to the action of applied stress is often neglected.

The purpose of this paper is to describe the development and performance of a macromodel for estimating creep and swelling, which utilizes existing mechanismbased models of microstructural evolution during irradiation and combines them in a fashion which allows for communication between them. This effort is intended to 
be a first attempt at developing a complete, self-consistent description of the evolution of creep and swelling in single crystal nickel under irradiation. The emphasis is, therefore, on the amalgamation of existing models into a macromodel and not on the individual processes themselves. The framework and methodology so constructed can, however, be used to evaluate individual mechanistic models.

\section{Background}

In order to formulate a self-consistent macromodel of creep and swelling, two basic components are required. The first is a complete description of the strain generating processes which occur at a given dose level. The second is a description of microstructural evolution which can be used to integrate the strain process over an irradiation history. Over the past several years, many investigators have made contributions to each of these areas, and preliminary work has been done on merging the two components together.

The initial development of microstructural modeling of strain generation was performed by Harkness, Tesk and $\mathrm{Li}$ [1] in 1969. These authors employed reaction rate theory and simplified nucleation models to simulate the evolution of void and interstitial loop distributions during fast neutron irradiation. Swelling rates were computed from the void behavior. Creep due to dislocation glide was calculated on the assumption that loops and voids served as dispersed barriers to glide. Creep due to the climb of dislocations was neglected and the dislocation density held constant.

Even in the absence of an evolving dislocation density, Harkness et al. were able to tune (through adjustable parameters) the model to compute swelling behavior rather accurately. The model generated irradiation creep rates which increased with dose rate, but decreased with increasing dose and temperature. The dose behavior is in direct conflict with experimental results published subsequently.

Three years later Wolfer, Foster and Garner [2] developed a similar approach but included some additional considerations. The dislocation glide model was modified to account for the stress exerted on the dislocation by loops acting as barriers. Creep strain contributions from dislocation climb and the preferential nucleation of loops were also added. As with Harkness' model, voids and loops were treated but no explicit account was made for the evolution of the network dislocation density. The authors reported a stress enhancement in swelling and glide-induced creep rate which is proportional to the swelling rate. Although no detailed evaluation was made, they reported that the creep rate due to preferential loop nucleation is in better agreement with measurements than that due to glide.

More recently, Weiner and Boltax [3] developed a microstructural model of creep and swelling which included dislocations, voids, interstitial loops and vacancy loops. The creep model includes contributions from the stress induced preferential absorption (SIPA) driven climb of dislocations and loop growth. Creep due to glide is entirely neglected. Void and loop nucleation models were empirically fitted to the data of Brager [11], but again there is no explicit account made of network dislocation evolution.

The calculated creep rates are found to be fairly insensitive to temperature below $450^{\circ} \mathrm{C}$ but rise rapidly as the temperature is increased to $550^{\circ} \mathrm{C}$ where they again level off at a higher value. The creep rate increases with dose as the loop population evolves and saturates when loop growth slows.

Aside from the projects discussed above, little else has been reported on the development of complete models. The bulk of the literature focuses on detailed descriptions of individual processes and mechanisms. Sophisticated computations have been made on the stress dependence of point defect capture rates [12-15], and the results have heen used to develop SIPA descriptions of creep $[4,7,9,10,12]$. Additional descriptions of creep due to dislocation glide have also been advanced $[5,8]$.

Recent efforts by Wolfer $[16,17]$ have lead to the development of models for network dislocation evolution. These models allow the computation of the dose dependent dislocation density and therefore its impact on dose dependent glide and climb strains. The incorporation of these models offers an opportunity to investigate microstructural relationships that could not be adequately evaluated when the early microstructural modeling was performed.

The computational model developed in this paper combines the rate theory of creep and swelling with the ability to track the evolution of the dislocation network in the attempt to develop a self-consistent description of dislocation evolution and its relation to the strain producing dislocation mechanisms.

\section{Macromodel development}

In single crystals, the dominant strain producing mechanisms are the climb and glide of dislocations. Dislocation glide is limited by the distribution of glide 
obstacles within the material and the manner in which the obstacles interact with glissile dislocations. Glide is also influenced by the rate of dislocation climb, which dictates the time required for dislocations to overcome an obstacle's pinning influence. Dislocation climb is governed by the rate at which the dislocation absorbs an excess of interstitials or vacancies. This differential absorption rate depends upon the relative preference of the dislocation for interstitials or vacancies and upon the concentrations of free interstitials and vacancies in the material. These considerations are in turn governed by the point defect production rates (damage rates), temperature dependent diffusion coefficients and the rates of defect absorption at other microstructural sinks.

Based on the physical description of important processes and relations given above, a model of microstructural development and strain generation is constructed as shown in fig. 1. The model operates on a quasi-steady state basis in which an irradiation history is divided into several time steps. Based on the microstructural information given at the beginning of a time step, defect concentrations and process rates are computed. These rates are assumed to be constant and act for the length of the time step $\Delta t$, at the end of which each variable is updated to its new value according to the relation $X(t+\Delta t)=X(t)+(\mathrm{d} X / \mathrm{d} t) \Delta t$. Concentrations and process rates are then recomputed based on the updated microstructure, and the procedure continues.

The goal of this project was to construct a macromodel that, while complete, was also self-consistent and simple. Toward this end, many of the geometric complexities of the real material were removed. In FCC nickel, dislocation loops tend to form on $\{111\}$ planes, and the dislocations responsible for glide are generally found on $\{111\},\langle 110\rangle$ slip systems [18]. Because the slip planes are not orthogonal, the application of stress or strain tensors to the system requires a complicated mathematical transformation. In order to simplify the situation, the orientation of loops and network dislocations was taken as being based on orthogonal cartesian planes rather than $\{111\}$ planes. This representation allows for a less complicated description of the material while preserving the fact that microstructural elements form in discrete orientations. A further simplication is made by neglecting the existence of faulted interstitial loops. The effect of this simplification is merely to limit the model's applicability to temperature regions where the loop density is low.

This section describes the individual process models which comprise the macromodel. Each of the models is expressed in terms of several material parameters. The

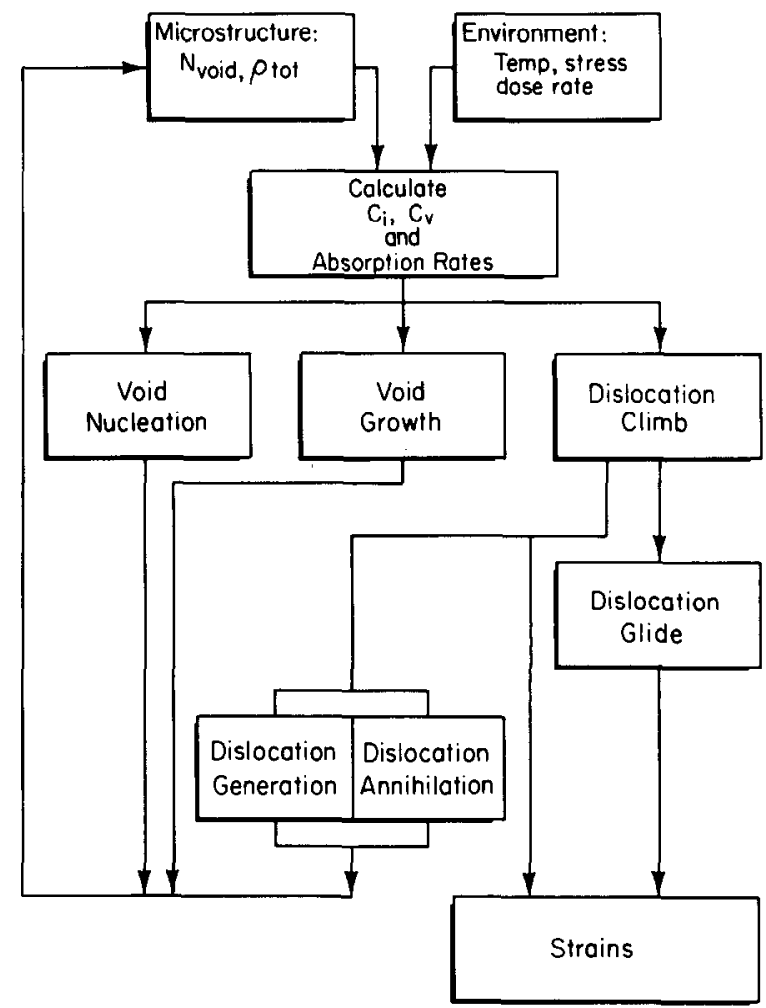

Fig. 1. A flowchart of the microstructural model of irradiation creep and swelling.

definitions and numerical values of these parameters are given in table 1 . Recall that the macromodel is intended to be a framework within which various descriptions of creep may be investigated. Individual creep models are treated as interchangeable modules and only one example is discussed in this section.

\subsection{Strain generation}

The macroscopic strains resulting from the climb and glide of edge dislocations may be expressed as a nonelastic strain tensor which may be decomposed into the sum of a dilatational component (swelling) and a distortional component (creep),

$$
\begin{aligned}
& {\left[\begin{array}{ccc}
\epsilon_{x x} & \epsilon_{x y} & \epsilon_{x z} \\
\epsilon_{y x} & \epsilon_{y y} & \epsilon_{y z} \\
\epsilon_{z x} & \epsilon_{z y} & \epsilon_{z z}
\end{array}\right]=\left[\begin{array}{ccc}
\epsilon_{\mathrm{v}} & 0 & 0 \\
0 & \epsilon_{\mathrm{v}} & 0 \\
0 & 0 & \epsilon_{\mathrm{v}}
\end{array}\right]} \\
& +\left[\begin{array}{ccc}
\left(\epsilon_{x x}-\epsilon_{\mathrm{v}}\right) & \epsilon_{x y} & \epsilon_{x z} \\
\epsilon_{y x} & \left(\epsilon_{y y}-\epsilon_{\mathrm{v}}\right) & \epsilon_{y z} \\
\epsilon_{z x} & \epsilon_{z y} & \left(\epsilon_{z z}-\epsilon_{\mathrm{v}}\right)
\end{array}\right],
\end{aligned}
$$


Table 1

Material parameters for nickel used in this study

\begin{tabular}{|c|c|c|c|}
\hline \multicolumn{4}{|c|}{ Constants: } \\
\hline Symbol & Definition & Value & Ref. \\
\hline $\bar{b}$ & Burgers vector & $2.57 \times 10^{10} \mathrm{~m}$ & [19] \\
\hline$\Omega$ & Atomic volume & $b^{3} / \sqrt{2}$ & - \\
\hline$a_{0}$ & Lattice constant & $b \sqrt{2}$ & - \\
\hline$D_{i}^{0}$ & Pre-exponential & $0.001 \mathrm{~m}^{2} / \mathrm{s}$ & [19] \\
\hline$D_{\mathrm{v}}^{0}$ & Pre-exponential & $0.100 \mathrm{~m}^{2} / \mathrm{s}$ & {$[20]$} \\
\hline$E_{\mathrm{i}}^{\mathrm{m}}$ & Int. migration energy & $0.2 \mathrm{eV}$ & [19] \\
\hline$E_{\mathrm{y}}^{\mathrm{m}}$ & Vac. migration energy & $1.4 \mathrm{eV}$ & {$[20]$} \\
\hline$E_{\mathrm{v}}^{\mathrm{F}}$ & Vac. formation energy & $1.8 \mathrm{eV}$ & [19] \\
\hline$\gamma$ & Surface energy & $2.0 \mathrm{~J} / \mathrm{m}^{2}$ & [21] \\
\hline$G$ & Shear modulus & $10^{11} \mathrm{~N} / \mathrm{m}^{2}$ & [21] \\
\hline$y$ & Poissons ratio & 0.3 & {$[21]$} \\
\hline$\alpha_{i}^{G}$ & Int. shear polarizability & $-150 \mathrm{eV}$ & [21] \\
\hline$\alpha_{\mathrm{v}}^{G}$ & Vac. shear polarizability & $-15 \mathrm{eV}$ & {$[21]$} \\
\hline$\alpha_{j}^{K}$ & Int. bulk polarizability & $100 \mathrm{eV}$ & [21] \\
\hline$\alpha_{y}^{K}$ & Vac. bulk polarizability & $-150 \mathrm{eV}$ & [21] \\
\hline K & Bulk modulus & $2.167 \times 10^{11} \mathrm{~N} / \mathrm{m}^{2}$ & [21] \\
\hline$u_{\mathrm{i}}$ & Int. relaxation volume & 1.4 & [21] \\
\hline$u_{\mathrm{v}}$ & Vac. relaxation volume & -0.2 & [21] \\
\hline$P$ & Void pressure & 0.0 & [21] \\
\hline$\epsilon_{i i}^{0}$ & Elastic dilatation strain & - & - \\
\hline$e_{i j}^{0}$ & Elastic deviatoric strain & - & - \\
\hline \multicolumn{4}{|c|}{ Functions: } \\
\hline \multicolumn{3}{|c|}{$\begin{array}{l}B_{0}=0.09095 u_{\alpha}^{K} \\
B_{1}=0.02274 \alpha_{\alpha}^{G} e_{y y}^{0}-0.09095 \alpha_{\alpha}^{K} \epsilon_{i j}^{0}-0.2046 \alpha_{\alpha}^{G} e_{x x}^{0} \\
A_{0}=2.07 \times 10^{-3} \alpha_{\alpha}^{K}+1.36 \times 10^{-2} \alpha_{\alpha}^{G}\end{array}$} & {$[12]$} \\
\hline
\end{tabular}

where $\Delta V / V=3 \epsilon_{\mathrm{v}}=\epsilon_{x x}+\epsilon_{y y}+\epsilon_{z z}$ is the swelling, and $\epsilon_{i j}^{\prime}=\epsilon_{i j}-\epsilon_{v} \delta_{i j}$ is the creep strain. The off diagonal elements of the tensor represent strains due to the glide of dislocations and the diagonal elements reflect dislocation climb. [22]:

Strain rates due to dislocation glide are expressed as

$\dot{\epsilon}_{i \neq j}=\rho b V_{\text {glide }}$,

where $\dot{\epsilon}$ is strain rate, $b$ is the magnitude of the Burgers vector, $V_{\text {glide }}$ is the glide velocity of the dislocations causing the shear strain, $\rho$ is the density of dislocations properly oriented to yield this strain component: $\hat{b} \| \hat{j}$, and on the $i$ th slip plane.

Strain rates due to dislocation climb are given by the expression [21]:

$\dot{\epsilon}_{i=j}=\rho b V_{\text {climb }}$,

where $\rho$ is the density of dislocations properly oriented to yields this strain component: $\hat{b} \| \hat{\jmath}, V_{\text {climb }}$ is the climb velocity of the dislocations causing the strain, $\dot{\epsilon}$ and $b$ are defined as above.

In order to compute the strain and strain rates, expressions for the climb and glide velocities of the network dislocations are needed.

\subsubsection{Dislocation glide rates}

Dislocation glide is treated by assuming that each dislocation is pinned at an obstacle for some length of time $\tau$. After $\tau$ has elapsed, the dislocation frees itself from the obstacles and glides, instantaneously, over the distance $l_{\mathrm{g}}$ to the next obstacle position where it will be pinned for another time $\tau$. The waiting time, $\tau$, is the time required for the dislocation to overcome its immediate obstacle. In the present model, dislocations may free themselves only be climbing away from the obstacle. Neither obstacle cutting nor dislocation bowing around obstacles (and pinching off) are treated. Given this simplification, $\tau$ becomes the climb distance, $h$, divided by the climb velocity, $V_{\text {climb. }}$. The glide veloc- 
ity may then be written as:

$V_{\text {glide }}=\left(l_{\mathrm{g}} / h\right) V_{\text {climb }}$.

Expressions for $l_{\mathrm{g}}$ and $h$ depend upon the treatment of obstacle spacing and the nature of the dislocation-obstacle interaction. The glide model used in the code is described by Olander [23] and attributed to Harkness [24]. In this model, dislocation glide is assumed to be impeded by an array of voids. As shown in fig. 2 , associated with each void is a lead dislocation which is assumed to be permanently pinned. Subsequent mobile dislocations must climb to a height sufficient to overcome the dislocation-dislocation repulsive force before gliding past the barrier. The effective barrier height $h$ is dependent on the applied shear stress through the expression:

$h=\frac{G b}{8 \pi(1-\nu) \sigma_{x y}}$.

The value of the glide distance, $l_{\mathrm{g}}$, is computed from the probability that a void will intersect a dislocation's slip plane. For voids forming a simple cubic structure,

$l_{\mathrm{g}}=\left(2 R_{\text {void }} N_{\text {void }}\right)^{-1 / 2}$.

\subsubsection{Dislocation climb rates}

The dislocation climb velocity is governed by the rate at which the dislocation absorbs an excess of either vacancies or interstitials. The climb velocity is related to the differential absorption rate by the relation [21]:

$V_{\text {climb }}=\frac{\Omega}{b} J_{\text {net }}$

where $J_{\text {net }}=J_{\text {int }}-J_{\text {vac }} \cdot V_{\text {climb }}$ is defined to be positive for $J_{\text {net }}>0$.

The net absorption rates for each type of defect sink (dislocations, voids, etc.) are calculated via steady state reaction rate theory. The absorption rates are expressed as the products of bulk average defect concentrations, $C$, and sink absorption rate constants, $k$. The defect concentrations are determined through the point defect balance equations. The equations may be expressed as [25]:

$P_{\mathrm{i}}-K_{\mathrm{i}} C_{\mathrm{i}}-\kappa C_{\mathrm{i}} C_{\mathrm{v}}=0$,

$\left(P_{\mathrm{v}}+\psi\right)-K_{\mathrm{v}} C_{\mathrm{v}}-\kappa C_{\mathrm{i}} C_{\mathrm{v}}=0$,

where

$P_{\alpha}$ is the production rate of the $\alpha$-type defect,

$C_{\alpha}$ is the concentration of the $\alpha$-type defect,

$K_{\alpha}$ is the total absorption rate constant for the $\alpha$-type defect, $K_{\alpha}=\sum_{j=\operatorname{sinks}} k_{\alpha}^{j}$,

$\kappa$ is the recombination coefficient, and
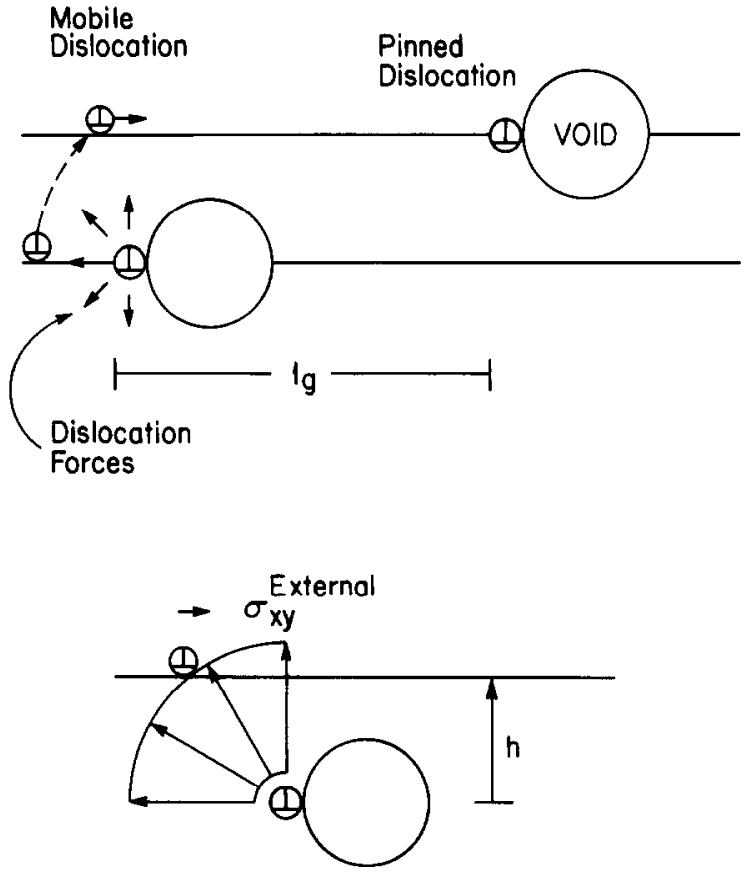

$$
h=\frac{G b}{8 \pi(1-\nu) \sigma_{x y}}
$$

Fig. 2. Dislocation glide model

$\psi$ is the thermal vacancy generation rate.

The treatment of these equations is described in many of the references cited earlier, specifically ref. [25]. As written, the balance equations do not account for the influence of point defect trapping. Because the model is applied only to pure single crystal metals, the effect of trapping is likely to be negligible.

It must be noted that the production rates $P_{\mathrm{i}}$ and $P_{\mathrm{v}}$ are the rates at which free interstitials and vacancies are produced by the damaging radiation. A value of 0.25 for the production efficiency was selected here in order to maximize agreement between void swelling measurements and preliminary computations. The true values of the production efficiency is poorly known and often used as a tuning parameter.

Another important consideration is that no account is made for self-ion injection during the irradiation. The inclusion of such an effect would raise $P_{\mathrm{i}}$ slightly relative to $P_{\mathrm{v}}$. This increase will impact most heavily on systems in which the point defect biases are low.

The recombination coefficient, $\kappa$, is expressed in its usual form as being proportional to the interstitial 
diffusion coefficient. The exact formulation is given by Wolfer [24].

$\psi$ is the thermal vacancy emission rate and is used to reflect the fact that at finite temperature there is a non-zero equilibrium concentration of vacancies in the material. There is also a non-zero concentration of interstitials but the value is so low as to be negligible [12]. Because the sample is treated as being infinite, surfaces are ignored and the only sources of thermally generated vacancies are the microstructural sinks. The total generation rate is given by the sum of generation rates at each sink. Each of these terms is written as the product of the vacancy rate constant for the sink and the vacancy concentration at the sink surface. The concentrations are taken to be in equilibrium with both the temperature and the stress state at the surface of the sink. This condition accounts for both thermal and stress driven vacancy emission. The exact formulations are given in refs. [23] and [24].

The rate constants $k^{j}$ are composed of three factors; a geometric factor, $G$, a capture efficiency, $Z$, and the defect diffusion coefficient, $D,[25]$

$k_{\alpha}^{j}=G^{j} Z_{\alpha}^{j} D_{\alpha}$.

The factors $G$ and $Z$ have been calculated via a number of varying techniques and these calculations have been reviewed at length by Nichols [27]. The factors used in this study were computed by Wolfer $[12,13,26]$.

In the case of voids and straight edge dislocations the geometric factors may be written,

$G_{\text {void }}=4 \pi R_{\text {void }}$,

$G_{\text {edge }}=2 \pi / \ln \left(R_{i} / a\right)$,

where $R_{\text {void }}$ is the void radius, $R_{1}$ is the radius of the outer boundary of the dislocation diffusion cell $\left(R_{\perp}=\right.$ $\left.0.5 \rho^{-1 / 2}\right), a$ is the inner cell boundary radius at which the defects are considered to be absorbed $(a=4 b)$, and $\rho$ is the dislocation density.

The capture efficiencies, written for either vacancies or interstitials depending on the choice of values for certain parameters $(\alpha=\mathrm{i}$ or $\mathrm{v})$, are expressed as $[12,13,28]$ :

$$
\begin{aligned}
& Z_{\alpha}^{\text {void }}=1-\frac{3}{56} \frac{\alpha_{\alpha}^{G}}{K T}\left(P-\frac{2 \gamma}{R_{\text {void }}}+K \epsilon_{i i}^{0}\right)^{2} / G^{2} \\
& +\left[0.463\left(\frac{G u_{\alpha}}{K T}\right)^{2}+0.0752 \frac{\alpha_{\alpha}^{K}}{K T}+2.75 \frac{\alpha_{\alpha}^{G}}{K T}\right] e_{i j}^{0} e_{i j}^{0}, \\
& Z_{\alpha}^{\text {disloc }}=1+\frac{1}{16 a^{2} \ln \left(R_{\perp} / a\right)}\left(\frac{B_{0}}{K T}\right)^{2}-\frac{8 A_{0}}{K T}+\frac{2 B_{0} B_{1}}{(K T)^{2}},
\end{aligned}
$$

where a value of 0.3 has been assumed for the clastic Poisson's ratio and the other parameters are given in table 1 . These expressions were selected because they are more complete than other treatments available in the literature. Simpler expressions are likely to take insufficient account of significant contributions to the sink strengths.

Having reviewed all of the models that directly pertain to the generation of strains, attention is turned to the models needed to quantify the evolution of the microstructure. Specifically, the nucleation of voids and the generation and annihilation of dislocations is discussed.

\subsection{Microstructure evolution}

\subsubsection{Void nucleation}

Numerous theoretical models of void nucleation have been proposed and developed [29,30]. Unfortunately, the current state of these models is such that they do not generate accurate descriptions of observed void number densities and show unrealistically large sensitivities to variations in certain parameters such as surface energy [20]. Because the mechanism-based nucleation models are so inaccurate, simple empirically derived void models are utilized, thus violating (out of necessity) the self-consistency criterion.

In this treatment, the void model was developed from data taken during self ion bombardment studies of solution annealed nickel [20]. The experimental conditions are described in table 2 . From the data, $N_{\text {void }}$ is related to temperature at a fixed dose, dose rate, starting microstructure and stress. This is shown in fig. 3 . The effects of other parameters are discussed below.

The variation of $N_{\text {void }}$ with dose is accounted for by assuming that all the voids observed are present at time zero, but as very small ( $15 \AA$ ) nuclęi; i.e., $N_{\text {void }}$ is taken to be constant in time. For an ion irradiation in which no helium is generated, this assumption is reasonably accurate. Whitley [31] observed the dose dependent void number density under similar experimental conditions and found that $N_{\text {void }}$ was virtually constant beyond 0.5 $\mathrm{dpa}$ for dose rates ranging from $5 \times 10^{-4}$ to $2 \times 10^{-3}$ $\mathrm{dpa} / \mathrm{s}$. This indicates that the voids nucleate only during the very early stages of irradiation.

The influence of stress on void density has been found to be significant. The correlation used in this study was developed from data supplied by Brager for solution annealed type 316SS [32]. The relation may be expressed,

$$
N_{\text {void }}(\sigma)=N_{\text {void }}(0) \exp \left(\frac{d \sigma_{\mathrm{H}}}{K T}\right)
$$


Table 2

Experimental conditions used for this study (ref. [20])

\begin{tabular}{ll}
\hline Material: & Nickel \\
Condition: & Solution annealed \\
Irradiation: & Self-ion \\
Dose rate: & $7 \times 10^{-4} \mathrm{dpa} / \mathrm{s}$ \\
Goal dose: & $13 \mathrm{dpa}$ \\
Temperature: & $300-625^{\circ} \mathrm{C}$ \\
Stress: & Unstressed \\
\hline
\end{tabular}

where $\sigma_{\mathrm{H}}$ is hydrostatic stress and $d$ is a fitting constant found to be $12.2 \Omega$ (from ref. [32]) where $\Omega$ is the atomic volume.

Dose rate and starting microstructure are also expected to influence the void nucleation behavior. Unfortunately, the available data base is not sufficiently populated to allow empirical correlations to be formulated with any degree of confidence. Since these influences are not accounted for, the nucleation model is limited to the starting microstructure and dose rate of the experiments from which the data were taken. These values are given in table 2 .

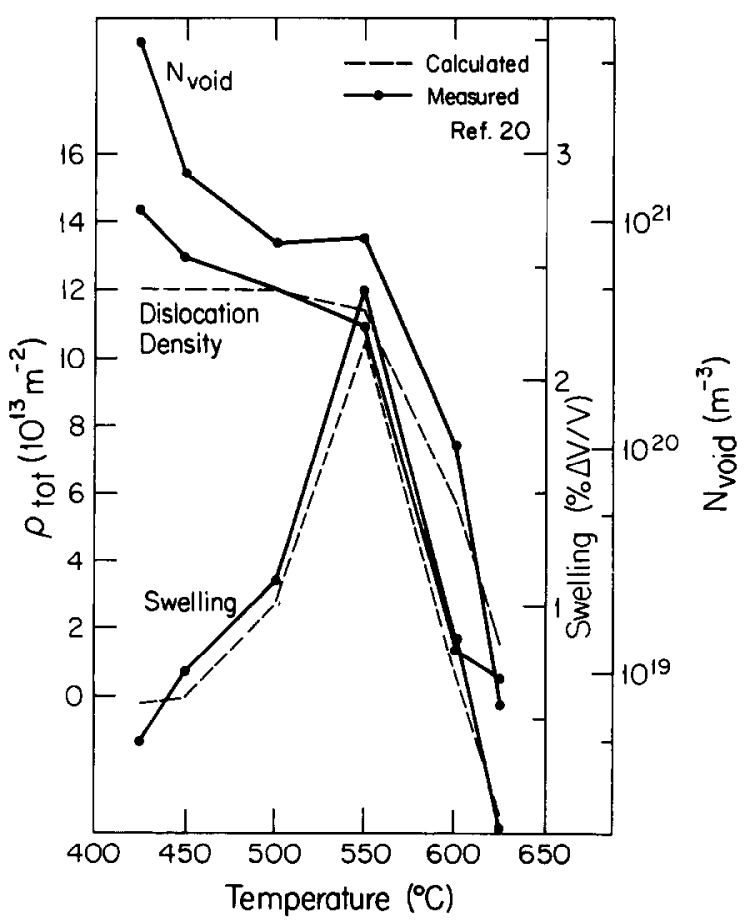

Fig. 3. Measured and computed values of dislocation density, void density and void swelling vs. temperature.
The final form of the void number model is expressed,

$N_{\text {void }}(\sigma, T)=N_{\text {void }}(0, T) \exp \left(\frac{d \sigma_{\mathrm{H}}}{K T}\right)$,

where the values of $N_{\text {void }}(0, T)$ are taken from fig. 3 for temperatures between 425 and $625^{\circ} \mathrm{C}$. Voids are assumed to grow from an initial radius of $15 \AA$. Although the value of the starting radius is arbitrary, it has little effect on the results. The void size distribution is therefore, a delta function. Processes which cause a spread in the distribution, such as coalescence are not treated in this model.

\subsubsection{Dislocation generation and annihilation}

The dose evolution of the network dislocation density results from competition between generation and annihilation mechanisms. This competition is expressed by Wolfer [17] as:

$\frac{\mathrm{d}}{\mathrm{d} t} \rho_{\text {net }}=B V_{\text {climb }} \rho_{\text {net }}-A V_{\text {climb }} \rho_{\text {net }}^{2}$,

where $A$ and $B$ are material parameters, dependent on the microstructure of the material, $V_{\text {climb }}$ is the dislocation climb velocity, and $\rho_{\text {net }}$ is the network dislocation density. Physically, the first term represents the generation of dislocation line length due to the bowing of pinned edge dislocations. The second term reflects annihilation due to dislocations of opposite sense climbing into close proximity. As illustrated by Wolfer, eq. (14) predicts that $\rho$ approaches a saturation value given by $\rho_{\text {sat }}=B / A$ and that the rate at which saturation is approached is governed by $B$. The dislocation model may be fit to experimental results but adjusting these two parameters.

For the present effort, the model was fit to experimental dislocation density values measured along with the void size measurements discussed earlier [20]. The temperature dependent, measured dislocation density is plotted as a solid line in fig. 3. These values were measured at $13.0 \mathrm{dpa}$ and are all assumed to be at equilibrium saturation levels. The flat region, between 425 and $550^{\circ} \mathrm{C}$ reflects the insensitivity of the network dislocation density to temperature. In this regime it is assumed that the bulk of the dislocation density can be attributed to edge dislocations and that loop line length may be ignored. As discussed by Garner [16], at temperatures where the thermal emission of defects may be taken as small $\left(<600^{\circ} \mathrm{C}\right)$ the network dislocation density is observed to be insensitive to temperature, but the line length attributed to loops maintain a strong temperature dependence. In fig. 3 , the relative insensitivity of total dislocation density to temperature (from 425 to 
$550^{\circ} \mathrm{C}$ ) suggests that over this range only a small part of the total could be due to loops. At higher temperatures, the drop in dislocation density may by due to thermal enhanced annihilation [17].

The ratio of $B / A, \rho_{\text {sat }}$, was set to $1.2 \times 10^{14} \mathrm{~m}^{-2}$ in order to match the experimental dislocation density values between 425 and $550^{\circ} \mathrm{C}$. The value of $B$ was set to $3.0 \times 10^{7} \mathrm{~m}^{-1}$ to match measurements of both dislocation density and void swelling. The constant was selected such that the dislocation density saturates by $13 \mathrm{dpa}$, and the temperature dependent swelling calculations agree with measurements. The value selected for $B$ was compared to the value expected from Wolfer's [17] theoretical model and found to be larger by a factor of 30 . The theoretical value of $B$ depends quite strongly on the distance between pinning centers responsible for dislocation bowing. Since Wolfer's treatment did not explicitly include voids as pinning centers, it is reasonable to assume that the theoretical value of $B$ will differ significantly from $B$ in a solid with a high void density. The dose dependent dislocation density is given in fig. 4 and the temperature dependent end-of-life (EOL) dislocation density and swelling are given in fig. 3 , respectively. The calculated values agree quite well with experiment.

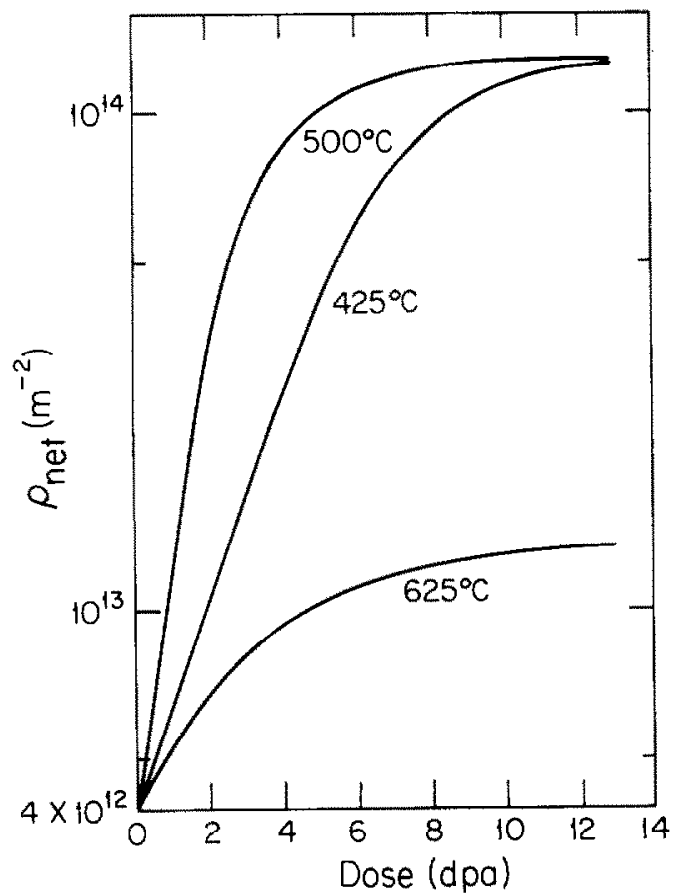

Fig. 4. Computed dose and temperature dependence of dislocation density.
With $N_{\text {void }}(T, \sigma)$ and $\rho(t)$ models set to the available data, the models are now tuned. The next step is to determine the response of the system to stress in order to calculate the creep behavior.

\subsection{Calculational methodology}

The models discussed above comprise the macromodel which is cast as a quasi-steady state computer code, illustrated in fig. 1. For a given time step, environmental and materials parameters are used to compute the terms in the point defect balance equations, eq. (8). This system is solved to yield the bulk average interstitial and vacancy concentrations. The defect concentrations are used to compute the dislocation climb velocities and void growth rates. The climb velocities along with microstructural information are input to the glide model which determines dislocation glide velocities. The climb and glide velocities are then used to determine the strain rate tensor via eqs. (1), (2) and (3). Finally, the climb velocities are input to the dislocation generation and annihilation model which computes $\mathrm{d} \rho / \mathrm{d} t$. This value, along with the void growth rate and the time step length are used to update the microstructure for the beginning of the next time step.

\section{Model performance}

The model is tuned using the set of conditions designated as base case \#1 in fig. 5. This parameter set serves as a zero-stress reference case. Base case $\# 2$ results from applying a stress to base case \#1 and serves as the reference case for performing creep calculations. The end-of-life (EOL) dose is $13 \mathrm{dpa}$.

The variables investigated were dislocation density, void number density, void radius, swelling strain and creep strain (due to climb and glide mechanisms). The parameters varied were dose, dose rate, temperature, stress and initial microstructure. A test matrix is given in fig. 5 .

\subsection{Effect of dose}

The dose dependence of creep, swelling and dislocation density was investigated for the case designated Base Case $\# 2$ and is shown in fig. 6. As expected, dislocation density increases during the early part of irradiation, approaching a saturation level at about 7 dpa. Void swelling increases with dose and the swelling rate also increases from zero to a value of $0.14 \% \Delta V / V$ per dpa after about $6 \mathrm{dpa}$. The swelling rate increases 


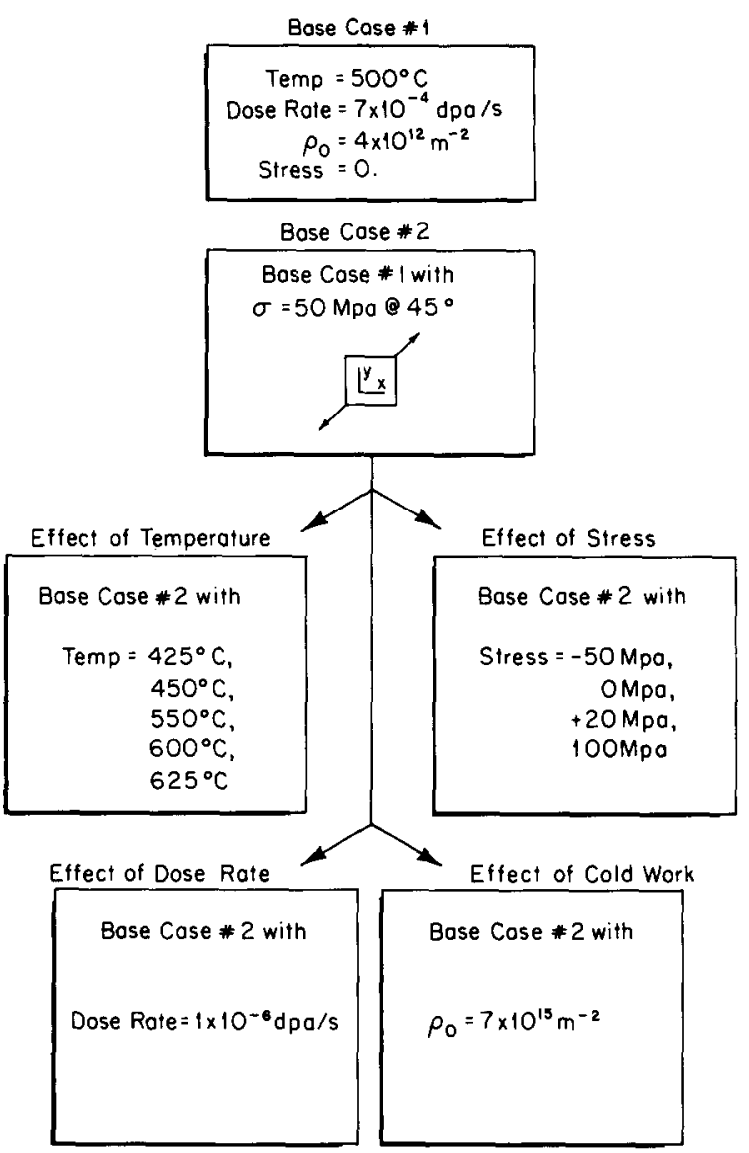

Fig. 5. A test matrix for evaluating model performance.

with $\rho$ and becomes constant once $\rho$ saturates. A linear dose dependence of void swelling has been observed by several experimenters $[3,33,34]$.

The creep strain also increases with dose in a fashion similar to that of void swelling. The creep curve becomes linear at low $(\approx 4 \mathrm{dpa})$ dose and assumes a creep rate of $0.083 \%$ per dpa. The linear dose dependence of steady state creep is widely observed in fcc metals $[26,35,36]$. The creep strain results almost entirely from dislocation glide rather than climb since the ratio of creep strain due to glide over that due to climb is more than three orders of magnitude.

Also plotted in fig. 6 is the dose dependence of void radius. As shown, the growth rate increases quickly at first and then levels off to a constant value as $\rho$ saturates. Near the end of life, the dose dependence becomes slightly less than linear. From the beginning to the end of life, the dose exponent of void radius changes from roughly 1.2 to 0.5 . As with swelling, the dose evolution

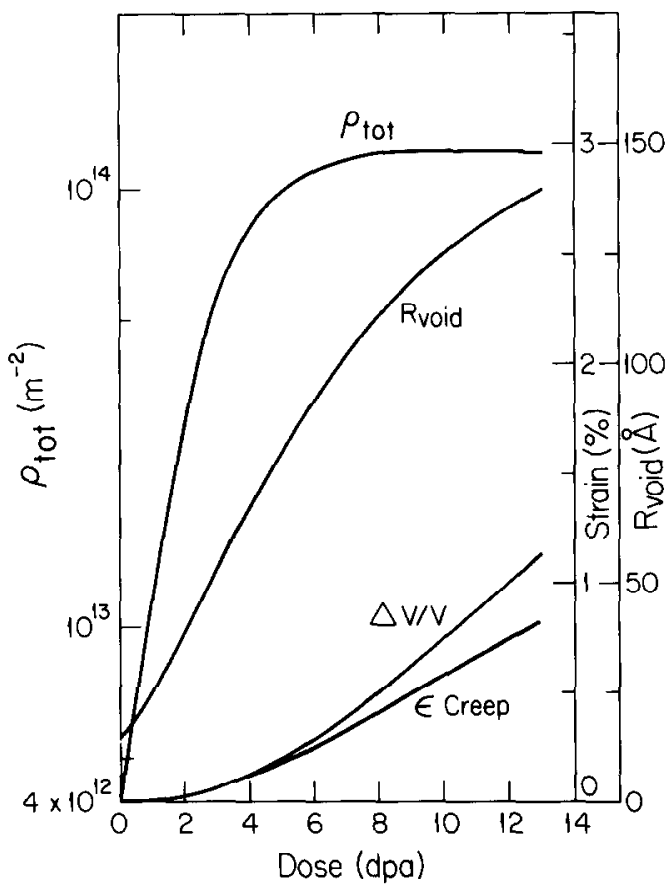

Fig. 6. Dose dependence of creep, swelling, dislocation density and void radius calculated for Base Case \#2.

of $R_{\text {void }}$ is consistent with measurements [31].

The most significant result of this case is the coupling of creep rate, swelling rate and void growth rate to the evolution of the dislocation density. The coupling is responsible for the initial increase in time of each of these rates. A model that did not include dislocation evolution would predict these rates as being constant in time.

\subsection{Effect of temperature}

The temperature dependence of EOL creep strain is plotted in fig. 7 for temperatures ranging from 425 to $625^{\circ} \mathrm{C}$. Also plotted here are the temperature profiles of void swelling, void number density and void radius. As shown, the temperature behavior of creep closely follows that of swelling. The temperature dependence of creep has not been definitively established experimentally and various authors report this dependence as being increasing [26] or decreasing [36,37].

Above the peak creep-swelling temperature, the decrease in creep and swelling strain with increasing temperature is due primarily to the decrease in dislocation density at these temperatures (see figs. 3 and 4). The reduced dislocation density may be related to the low 


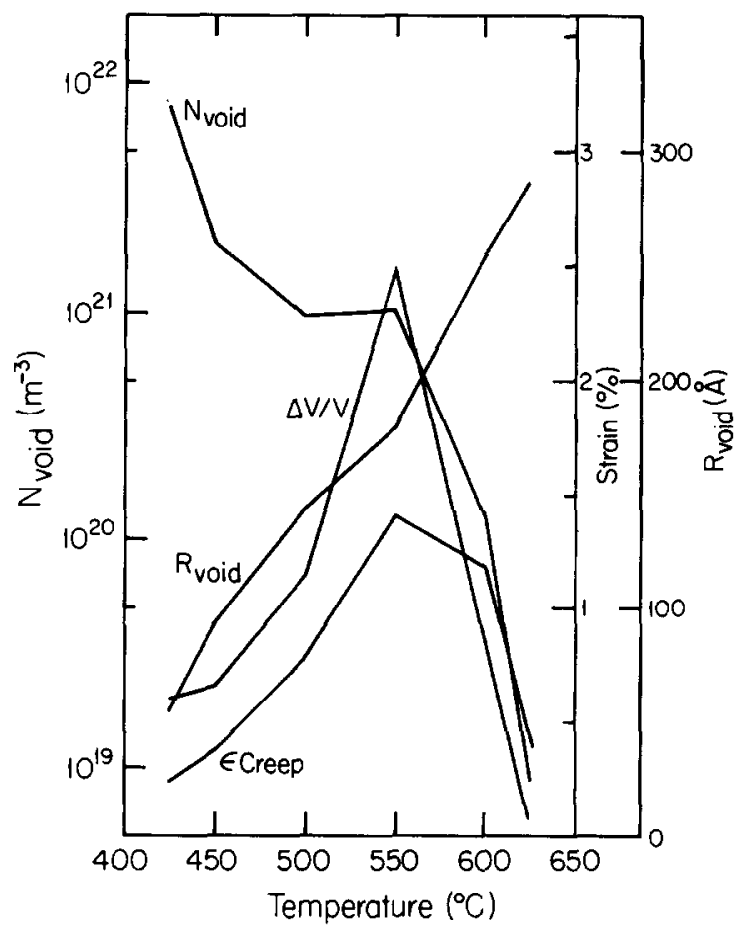

Fig. 7. Temperature dependence of creep, swelling, void number and void radius calculated for Base Case $\# 2$.

void number density which exists at higher temperatures. Since few voids develop, the driving force for point defect migration is retarded and subsequently, dislocation climb is slowed. The reduction in climb rate prevents the dislocation density from evolving to appreciable levels and the creep and swelling rates remain low. Without the interplay between microstructural evolution and strain generation, the predicted behavior could be drastically different.

\subsection{Effect of microstructure}

The effect of starting microstructure was investigated by performing a simulation similar to Base Case $\# 2$ but with an initial dislocation density of $7 \times 10^{15} \mathrm{~m}^{-2}$. The results of this run are plotted in fig. 8 . It should be recalled that since the void nucleation model was developed solely for the solution annealed case, it is not expected to adequately describe the behavior of cold worked material. As such, the investigation of microstructural effects must be considered preliminary.

As expected, the dislocation density decreased with dose from the initial state to the saturation level found in Base Case \#2. As in Base Case \#2, the change took

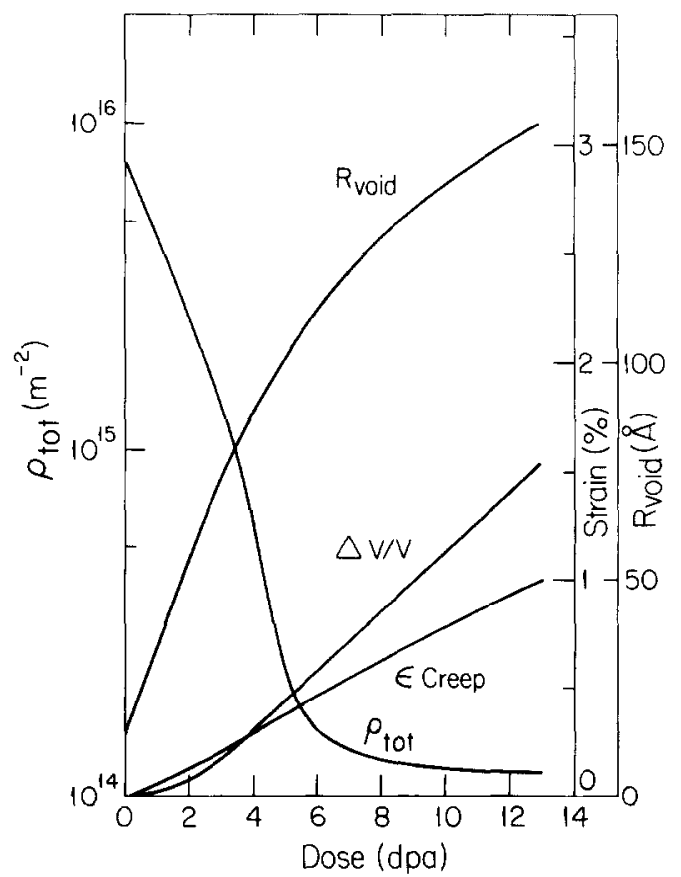

Fig. 8. Calculated dose dependence of creep, swelling, dislocation density and void radius for large initial dislocation density.

place over the first $7 \mathrm{dpa}$. Once the dislocation saturation level has been reached, swelling and creep rates attain values very near those of Base Case $\# 2$.

Early in life, as $\rho$ is decreasing, both swelling and creep rates are higher than in Base Case \#2 and saturate more quickly. This indicates that peak swelling and creep conditions exist for a dislocation density slightly higher than the saturation level of 'Base Case \#2. EOL swelling and creep strains are higher in the case of a high dislocation density but this is thought to be attributable to the inadequacy of the void nucleation model. In practice, cold work generally postpones void nucleation [33] and hence the curves would be shifted to the right, reducing EOL strains.

\subsection{Effect of dose rate}

The effect of dose rate was investigated by running a simulation similar to Base Case $\# 2$ but with a dose rate of $1 \times 10^{-6} \mathrm{dpa} / \mathrm{s}$ (fig. 9). This rate, 700 times lower than used previously, is considered typical of fast reactor (EBR-II) irradiation [38]. Again, it is recalled that since the model was not designed to treat the low dose rate case, results must be interpreted with care. Creep, swelling and dislocation evolution all proceed much 
faster (per dpa) at the lower dose rate. Relative to Base Case \#2, the swelling and creep rates $\left(\mathrm{dpa}^{-1}\right)$ are enhanced by factors of 16.5 and 19.4 respectively. The dislocation density approaches its saturation value in less than $1 \mathrm{dpa}$ instead of $7 \mathrm{dpa}$ and the saturation level is slightly lower than in Base Case \#2. Although the specific numerical values of this run may not be significant, a key result is that all process rates (when expressed per dpa) are much higher at the lower dose rate. This result is seen to stem from the fact that as dose rate (defect production rate) is increased, the fraction of defects destined for mutual annihilation (and therefore not for absorption at sinks) increases. The fraction of defects which migrate to sinks, causing the microstructure to evolve, decreases with increasing dose rate. Therefore, when the rates are expressed per unit dose (per unit defect produced) they are seen to decrease with increasing dose rate.

An inverse relation between strain rates and dose rates has been experimentally observed in a number of instances. Foster [35] found that when comparing creep rates between fast and thermal reactor experiments that, per fast neutron, creep rates in the thermal spectra were

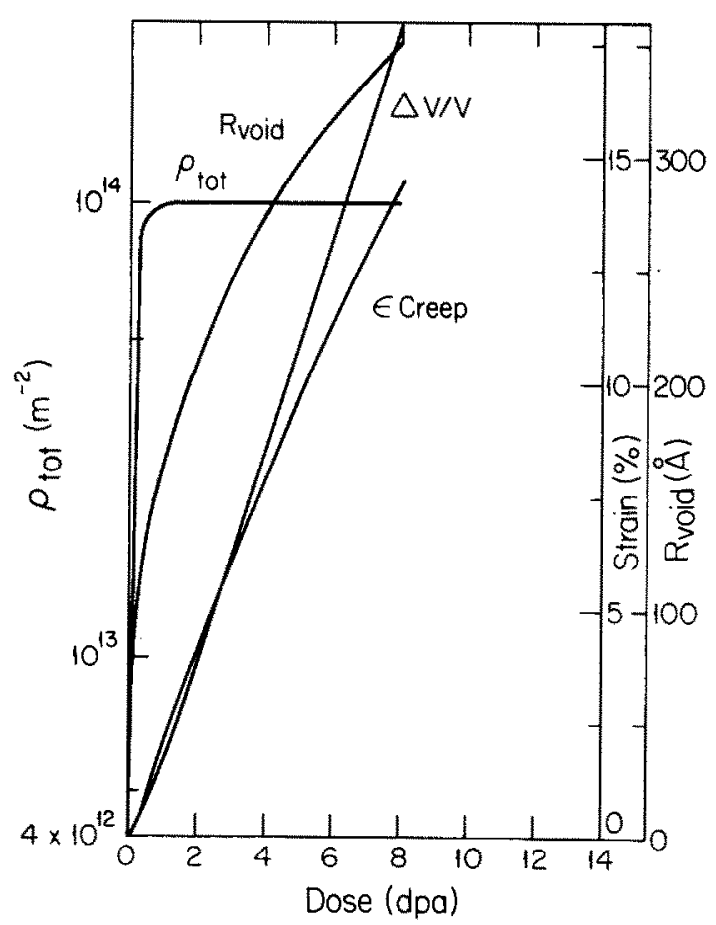

Fig. 9. Calculated dose dependence of creep, swelling, dislocation density and void radius for low dose rate. consistently 2.5 times higher than those measured in the fast spectra. Lewthwaite and Mosedale [39] also obtained an inverse relation when relating creep rate to dose rate in various fast reactor spectra. A similar dose rate dependence was observed in the swelling behavior of self-ion irradiated nickel [20]. At their respective "peak-swelling" temperatures, samples irradiated at 7 $\times 10^{-4} \mathrm{dpa} / \mathrm{s}$ displayed a larger swelling rate (per dpa) than those irradiated at $7 \times 10^{-2} \mathrm{dpa} / \mathrm{s}$.

\subsection{Effect of stress}

The effect of stress was investigated over the range of -50 to $+100 \mathrm{Mpa}$ (tensile) applied along the [110] direction. The response of EOL creep, swelling and void behavior is plotted in fig. 10. The dose dependent dislocation density was not noticeably altered and therefore not plotted.

As expected, void number density and void swelling are both enhanced by tensile stress. This result is in agreement with Ehrlich [33] who reports that swelling increases linearly with hydrostatic stress. Void radius is a decreasing function of tensile stress; the decrease in size resulting from the fact that a constant number of

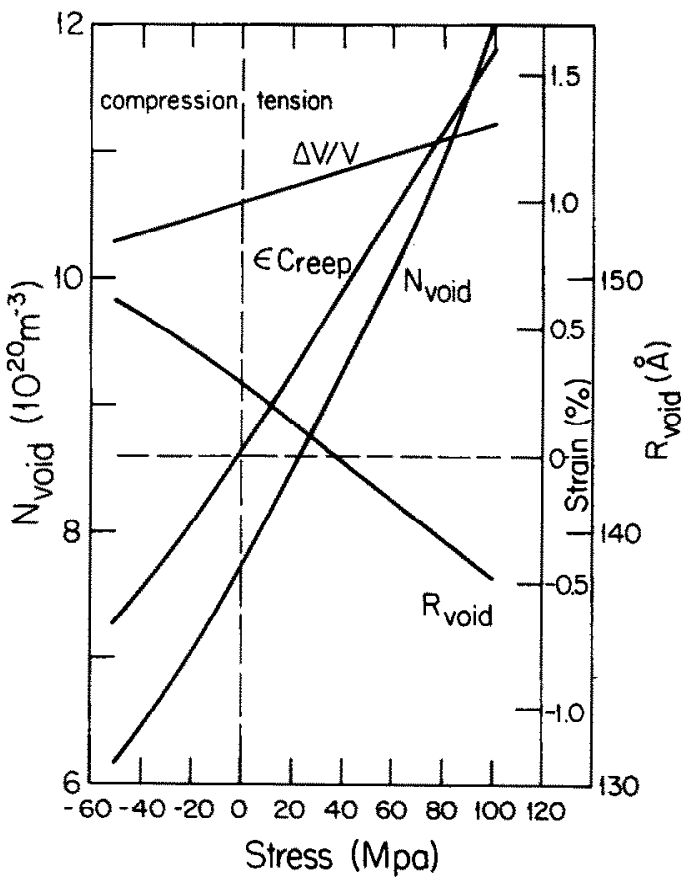

Fig. 10. Calculated stress dependence of creep, swelling, void number and void radius. 
Table 3

Creep coefficients for the glide model

\begin{tabular}{ll}
\hline $\begin{array}{l}\text { Temp. } \\
\left({ }^{\circ} \mathrm{C}\right)\end{array}$ & $\begin{array}{l}\mathrm{C} \\
\left(\mathrm{Mpa}^{-1} \mathrm{dpa}^{-1}\right)\end{array}$ \\
\hline 425 & $3.4 \times 10^{-6}$ \\
450 & $5.8 \times 10^{-6}$ \\
500 & $11.8 \times 10^{-6}$ \\
550 & $21.7 \times 10^{-6}$ \\
600 & $18.3 \times 10^{-6}$ \\
625 & $6.6 \times 10^{-6}$ \\
\hline
\end{tabular}

vacancies are being distributed among a larger number of voids when stress is applied. With fewer vacancies available to each void, growth is slower. For a constant number density, tensile stress is expected to accelerate void growth [40].

The steady state irradiation creep strain also increases linearly with stress as expected. As illustrated in fig. 10, there is a slight difference in slope from the compressive to the tensile side of the plot. If the only effect of stress on the system were on $h$ through eq. (5), then $\epsilon(\sigma)=-\epsilon(-\sigma)$ and no change in slope would occur. However, there is also a stress effect on the glide distance, $l_{\mathrm{g}}$, through its dependence of $N_{\text {void }}$ and $R_{\text {void }}$ (eq. (6)) and it is this effect which is responsible for the change in slope in fig. 10.

Given that steady state creep strain is determined to be linear in both dose and stress, a "creep coefficient" may be computed. This coefficient is defined as strain per unit dose and unit stress and is useful for comparing various creep correlations. As shown in table 3, the creep coefficients computed with the glide model range from $3.4 \times 10^{-6}$ to $21.7 \times 10^{-6} \mathrm{dpa}^{-1} \mathrm{Mpa}^{-1}$ over the temperatures investigated. These results correspond to the simulation of solution annealed, single crystal nickel under a self-ion dose rate of $7 \times 10^{-4} \mathrm{dpa} / \mathrm{s}$. Unfortunately, no experimental creep coefficient could be obtained for similar conditions and hence a direct assessment of the model's accuracy cannot be made.

Creep coefficients have, however, been measured for polycrystalline nickel under a variety of experimental conditions [35,36,41-43] and it is somewhat instructive to compare these values to the results obtained above. The available creep coefficients measured for nickel span a range from $8 \times 10^{-6}$ (ref. [36]) to $375 \times 10^{-6}$ (ref. [42]) $\mathrm{dpa}^{-1} \mathrm{Mpa}^{-1}$. Although these values include both neutron and ion irradiations, all these data were taken at temperatures and dose rates significantly lower than those studied with the present model. In addition, the measurements reflect a wider variety of material conditions and stress states. The computed results are lower than the measured coefficients, but the values are within an order of magnitude. As discussed below, the fact that the computed values are lower than the measurements can in part be explained by the fact that higher dose rates were used in the simulations.

The fact that the computed creep coefficients are in reasonable agreement with measurements indicates that the glide model is a fairly good description of events occurring in the real material assuming that the simplifjcation in crystal structure is appropriate. The glide model is based on the premise that voids are the dominant pinning centers, and that alternate centers such as dislocation network intersections may be ignored. This assumption may be supported, at least in these cases, by comparing the glide distance that result from taking either voids or dislocation intersections as the controlling pinning centers. From eq. (6),

$l_{\mathrm{g}}^{\text {void }}=\left(2 R_{\text {void }} N_{\text {void }}\right)^{-1 / 2}$.

For an intersection controlled model, the glide distance

Table 4

Ratio of dislocation intersection-controlled to void-controlled glide distances

\begin{tabular}{|c|c|c|c|c|c|}
\hline $\begin{array}{l}\text { Temp. } \\
\left({ }^{\circ} \mathrm{C}\right)\end{array}$ & $\begin{array}{l}\rho^{\text {a) }} \\
\left(10^{12} \mathrm{~m}^{-1}\right)\end{array}$ & $\begin{array}{l}N_{\text {vold }}{ }^{a)} \\
\left(10^{18} \mathrm{~m}^{-3}\right)\end{array}$ & $\begin{array}{l}R_{\text {yoid }}^{\text {a) }} \\
\left(10^{-10} \mathrm{~m}\right)\end{array}$ & $\left(\frac{l_{\mathrm{g}}^{\mathrm{disc}}}{l_{\mathrm{g}}^{\text {void }}}\right)$ & b) \\
\hline 425 & 143 & 6100 & 40 & 101 & \\
\hline 450 & 130 & 1600 & 100 & 83 & \\
\hline 500 & 120 & 770 & 145 & 77 & \\
\hline 550 & 110 & 830 & 185 & 91 & \\
\hline 600 & 15 & 100 & 275 & 105 & \\
\hline 625 & 4 & 7 & 150 & 40 & \\
\hline
\end{tabular}

\footnotetext{
2) From ref. [20].

b) From eqs. (15) and (16).
} 
is computed to be [44]:

$l_{\mathrm{g}}^{\mathrm{disc}}=\sqrt{3 / \rho}$.

Using the temperature dependent values of $R_{\text {void }}$ and $N_{\text {void }}$ from table $4, l_{\mathrm{g}}^{\text {void }}$ and $l_{\mathrm{g}}^{\text {disc }}$ are easily computed and their ratio is listed in the last column in table 4. For all temperatures considered, the void controlled glide distance is significantly shorter than the glide distance found for intersection control. This comparison supports the initial assumption that glide is void controlled. It should be noted, however, that in comparison to nickel, structural materials such as stainless steel generally display a larger dislocation density and a smaller void population. As such the void control assumption may not be entirely applicable to these situations.

\section{Conclusions}

A computational macromodel has been developed to estimate creep strain, swelling and microstructure evolution in single crystal nickel during irradiation. The model combines the rate theory of creep and swelling with the ability to track the evolution of the dislocation network. The macromodel serves as a first attempt to devclop a self-consistent description of dislocation evolution and its relation to the strain producing dislocation mechanisms.

The importance of the coupling of microstructural evolution to strain generation has been illustrated by the computer experiments presented in the text. The coupling is directly responsible for the following results:

- The "steady state" creep rate increases with dose from zero to its ultimate value. The increase results from the changes in dislocation density with dose.

- At high temperatures, crecp strain decreases with increasing temperature. The decrease is related to a reduced dislocation density which is caused by the lower void number density that exists at higher temperature.

- Steady state creep is linear in stress, but there is a discontinuity in slope at $\sigma=0$. The discontinuity results from the influence of stress on the void number density.

None of these results would have been predicted if the microstructural evolution had not been tracked. It is clear that the explicit coupling of microstructural evolution to the strain producing models is necessary if all the important features of the mechanisms are to be revealed.

The greatest deficiency of the present macromodel is the isolation of void nucleation from the rest of the code. To develop a completely self-consistent model, void nucleation must be included as a rate equation and integrated in concert with the other models.

\section{References}

[1] S.D. Harkness, J.A. Tesk and Che-Yu Li, Nucl. Appl. Tech. 9 (1970) 24.

[2] W.G. Wolfer, L.P. Foster and F.A. Garner, Nucl. Tech. 16 (1972) 55.

[3] R.A. Weiner and A. Boltax, J. Nucl. Mater. 66 (1977) 1.

[4] G.W. Lewthaite, J. Nucl. Mater. 79 (1979) 180.

[5] L.K. Mansur and T.C. Reiley, J. Nucl. Mater. 90 (1980) 60.

[6] C.H. Woo, Philos. Mag. A42 (1980) 551.

[7] A.I. Ryazanov and V.A. Borodin, Radiat. Eff. 59 (1981) 13.

[8] B.A. Chin, J.L. Strallsund and E.R. Gilbert, J. Nucl. Mater. 90 (1980) 68.

[9] P.T. Heald and J.E. Harbottle, J. Nucl. Mater. 67 (1977) 229.

[1.0] R. Bullough and M.R. Hayns, J. Nucl. Mater. 57 (1975) 348.

[11] H.R. Brager and J.L. Straasland, J. Nucl. Mater. 46 (1973) 134.

[12] W.G. Wolfer and M. Ashkin, J. Appl. Phys. 47 (1976) 791.

[13] W.G. Wolfer and M. Ashkin, J. Appl. Phys. 46 (1975) 547 (Note erratum: J. Appl. Phys. 46 (1975) 4108).

[14] R. Bullough, D.W. Wells, J.R. Willis and M.H. Wood, the interaction energy between interstitial atoms and dislocations and its relevance to irradiation damage processes, in: Dislocation Modelling of Physical Systems, Eds. M.F. Ashby, R. Bullough, C.S. Hartley, J.P. Hirth, conf. Proc. Gainesvill, Florida, June 1980, p. 116.

[15] W.A. Coghlan and M.H. Yoo, Radius dependence of the sink strength of a dislocation loop, ibid. ref. [14], p. 152.

[16] F.A. Garner and W.G. Wolfer, A Model for the evolution of network dislocation density in irradiated metals, in: Proc. Eleventh Conf. on Effects of Radiation on Materials, ASTM STP 782, Eds. H.R. Brager and J.S. Perrin, ASTM, STP 782, (ASTM, 1982) p. 1073.

[17] W.G. Wolfer, B.B. Glasgow and Ji Jung Kai, A rate-theory model for evolution of network dislocations in irradiated metals, in: Damage Analysis and Fundamental Studies: Quarterly Progress Report, DOE, (May 1984) p. 38.

[18] R.E. Reed-Hill, Physical Metallurgy Principals (Brooks/ Cole Engineering Division, Monterey, California, 1973) Chapter 4.

[19] W.G. Wolfer and B.B. Glasgow, Growth mechanisms for helium inclusions and bubbles, in: Damage Analysis and Fundamental Studies: Quarterly Progress Report, DOE, (February 1983) p. 115.

[20] J.E. Westmoreland, J.A. Sprague, F.A. Smidt and P.R. Malberg, Radiat. Eff. 26 (1975) 1.

[21] W.G. Wolfer, M. Ashkin and A. Boltax, Creep and swell- 
ing deformation in structural matcrials during fast-neutron irradiation, in: Properties of Reactor Structural Alloys After Neutron or Particle Irradiation, ASTM STP 570 (ASTM, 1975) p. 233.

[22] D.R. Olander, Fundamental Aspects of Nuclear Reactor Fuel Elements (Technical Information Center, DOE, 1976) Chapter 8.

[23] D.R. Olander, Fundamental Aspects of Nuclear Reactor Fuel Elements (TEchnical Information Center, DOE, 1976) Chapter 19.7.4.

[24] S.D. Harkness, R. Grappel and S.G. McDonald, Nucl. Tech. 16 (1972) 25.

[25] L.K. Mansur, Nucl. Tech. 40 (1978) 5.

[26] W.G. Wolfer, J. Nucl. Mater. 90 (1980) 175.

[27] F.A. Nichols, Radiat. Eff. 39 (1978) 169.

[28] W.G. Wolfer, L.K. Mansur and J.A. Sprague, Theory of swelling and irradiation creep, in: Radiation Effects in Breeder Reactor Structural Materials, Eds. M.L. Bleiberg and J.W. Bennett (Scottsdale Az., June 1977). p. 841.

[29] K.C. Russcll, Acta Metall. 19 (1971) 753.

[30] R.W. Powell and K.C. Russell, Radiat. Eff. 12 (1972) 127.

[31] J.B. Whitley, G.L. Kulinski, P. Wilkes and J. Billen, Depth dependent swelling rates in self-ion irradiated nickel, UWFDM-284, Univ. of Wisconsin (1979).

[32] H.R. Brager, F.A. Garner, E.R. Gilbert, J.E. Flinn and W.G. Wolfer, Stress-affected microstructural development and the creep-swelling interrelationship, ibid. ref. [21], p. 727.
[33] K. Ehrlich, J. Nucl. Mater. 100 (1981) 149.

[34] J.E. Harbottle and S.M. Dickerson, J. Nucl. Mater. 44 (1972) 313.

[35] J.P. Foster and A. Boltax, J. Nucl. Mater. 89 (1980) 331.

[36] A.R. Causey, G.J.C. Carpenter and S.R. MacEwen, J. Nucl. Mater. 90 (1980) 216.

[37] W.N. McElroy, R.E. Dahl, Jr. and E.R. Gilbert, Nucl. Engng. Des. 14 (1970) 319.

[38] D.R. Olander, Fundamental Aspects of Nuclear Reactor Fuel Elements (Technical Information Center, DOE, 1976) Chapter 17.

[39] G.W. Lethwaite and D. Mosedale, J. Nucl. Mater. 90 (1980) 205.

[40] F.A. Garner, W.G. Wolfer, A. Biancheria and A. Boltax, The effect of stress on radiation-induced void growth, in: Proc. Radiation-induced Voids in Metals, Eds. J.W. Corbett and L.C. Ianniello, Albany, NY, June 1971, p. 841.

[41] P.L. Hendrick, D.J. Michel, A.G. Piper and R.E. Surratt, J. Nucl. Mater. 59 (1976) 229.

[42] E.P. Simonen and C.H. Henager, J. Nucl. Mater. 103\&104 (1981) 1281.

[43] R.B. Jones and I.G. Crossland, The influence of alloying on the irradiation creep of nickel, ibid. ref. [21], p. 443.

[44] D.R. Olander, Fundamental Aspects of Nuclear Reactor Fuel Elements (Technical Information Center, DOE, 1976) Chapter 13.12. 